\title{
FINITE GROUPS WITH FEW CHARACTER VALUES
}

\author{
SESUAI Y. MADANHA
}

\begin{abstract}
A classical theorem on character degrees states that if a finite group has fewer than four character degrees, then the group is solvable. We prove a corresponding result on character values by showing that if a finite group has fewer than eight character values in its character table, then the group is solvable. This confirms a conjecture of T. Sakurai. We also classify non-solvable groups with exactly eight character values.
\end{abstract}

\section{INTRODUCTION}

Let $G$ be a finite group and $\operatorname{Irr}(G)$ be the set of complex irreducible characters of $G$. Recall the definition of a character degree set:

$$
\operatorname{cd}(G):=\{\chi(1) \mid \chi \in \operatorname{Irr}(G)\} .
$$

The study of the set $\operatorname{cd}(G)$ has received much attention from many authors and has been shown to have strong influence on the structure of $G$. In particular, we have the famous Ito-Michler theorem which states that $G$ has a normal abelian Sylow $p$ subgroup if and only if $p$ does not divide any member of $\operatorname{cd}(G)$. On the other extreme is Thompson's theorem, which states that if $p$ divides every member of $\operatorname{cd}(G) \backslash\{1\}$, then $G$ has a normal $p$-complement. In this note we shall study a bigger set found in a character table:

$$
\operatorname{cv}(G)=\{\chi(g) \mid \chi \in \operatorname{Irr}(G), g \in G\} .
$$

Little has been done in the study of this set. The first article in this direction is from T. Sakurai [8], who studied groups with very few character values. We shall compare results in [8] with corresponding character degree results. It is well known that $|\operatorname{cd}(G)|=1$ if and only if $G$ is abelian. Sakurai showed that if $|\operatorname{cv}(G)| \leq 3$, then $G$ is abelian. The converse is not true since $\left|\operatorname{cv}\left(\mathrm{C}_{r}\right)\right|=r$, where $\mathrm{C}_{r}$ denote the cyclic group of order $r$ for some positive integer $r$.

If $|\operatorname{cd}(G)|=2$ with $\operatorname{cd}(G)=\{1, n\}$, then either $G$ has an abelian normal subgroup of index $n$, or $n=p^{e}$ for a prime $p$ and $G$ is direct product of a $p$-group and abelian group. It turns out that a non-abelian group $G$ is such that $|\operatorname{cv}(G)|=4$ if and only if $G$ is the generalized dihedral group of a non-trivial elementary abelian 3-group ( 8 , Theorem]). A generalized dihedral group of a non-trivial elementary abelian $p$-group is a group $G_{n}=\left[\mathrm{C}_{p} \times \mathrm{C}_{p} \times \cdots \times \mathrm{C}_{p}\right] \rtimes \mathrm{C}_{2}$, the semidirect product of the direct product of $n$ copies of $\mathrm{C}_{p}$ and $\mathrm{C}_{2}$, where $\mathrm{C}_{2}$ acts on $\left[\mathrm{C}_{n} \times \mathrm{C}_{n} \times \cdots \times \mathrm{C}_{n}\right]$ without non-trivial fixed points. For groups with more than four character values, Sakurai remarked on the difficulty to classify these groups. However, a conjecture was proposed which states that if the group has fewer than eight character values, then the group is solvable [8, Remark]. Our aim is to settle this conjecture:

Date: June 30, 2021.

2010 Mathematics Subject Classification. Primary 20C15.

Key words and phrases. character values, character degrees, almost simple groups. 
Theorem 1.1. If $|\mathrm{cv}(G)|<8$, then $G$ is solvable.

Isaacs [5, Theorem 12] proved a result that is now considered classical which states that if $|\operatorname{cd}(G)|<4$, then $G$ is solvable. Hence Theorem 1.1 is the corresponding result for character values. Our proof uses the classification of finite simple groups.

Malle and Moretó [7] classified non-solvable groups with $|\operatorname{cd}(G)|=4$ (see Theorem 2.2). We obtain a corresponding result for character values:

Theorem 1.2. Let $G$ be a finite non-solvable group. Then $|\operatorname{cv}(G)|=8$ if and only if $G \cong \mathrm{PSL}_{2}(5)$ or $\mathrm{PGL}_{2}(5)$.

\section{Preliminary Results}

In this section we list some preliminary results that we need to prove our main results. We shall use freely these well known results in the following lemma.

Lemma 2.1. Let $G$ be a finite group, $N$ be a normal subgroup of $G$ and $n$ be a positive integer. Then the following hold:

(a) $|\mathrm{cv}(G / N)| \leq|\mathrm{cv}(G)|$,

(b) The character table of $\mathrm{C}_{n}$ has a column with pairwise different values. In particular, $\left|\mathrm{cv}\left(\mathrm{C}_{n}\right)\right|=n$,

(c) If $G$ is non-abelian, then $0 \in \mathrm{cv}(G)$.

The following is a result of Malle and Moretó [7] in which they classified non-solvable groups with four character degrees.

Theorem 2.2. [7, Theorem A] Let $G$ be a non-solvable group with $|\operatorname{cd}(G)|=4$. Then one of the following holds:

(a) $G \cong \mathrm{PSL}_{2}\left(2^{f}\right) \times A$ for some $f \geq 2$ and some abelian subgroup $A$;

(b) $G$ has a normal subgroup $U$ such that $U \cong \operatorname{PSL}_{2}(q)$ or $\operatorname{SL}_{2}(q)$ for some odd $q \geq 5$, and if $C=C_{G}(U)$, then $C \leq \boldsymbol{Z}(G)$ and $G / C \cong \mathrm{PGL}_{2}(q)$; or

(c) the group $G$ has a normal subgroup of index 2 that is a direct product of $\operatorname{PSL}_{2}(9)$ and central subgroup $C$. Furthermore, $G / C \cong M_{10}$, the Mathieu group which the stabilizer of a point in the Mathieu group, $M_{11}$, in its natural permutation representation.

Conversely, if one of (a)-(c) holds, then $|\operatorname{cd}(G)|=4$.

He and Zhu [4] described non-solvable groups with five and six character degrees:

Theorem 2.3. [4, Corollary C] Let $G$ be a non-solvable group and $L$ be the solvable radical of $G$. If $5 \leq|\operatorname{cd}(G)| \leq 6$, then $G^{\prime} / L$ is isomorphic to $\operatorname{PSL}_{2}\left(p^{f}\right)$ with $p^{f} \geq 4$, $\mathrm{PSL}_{3}(4)$ or ${ }^{2} \mathrm{~B}_{2}\left(2^{f}\right)$, where $2^{f}=2^{2 m+1}$ for some integer $m \geq 1$. Moreover, $G / L$ is an almost simple group.

We classify almost simple groups with five character degrees. We first have some

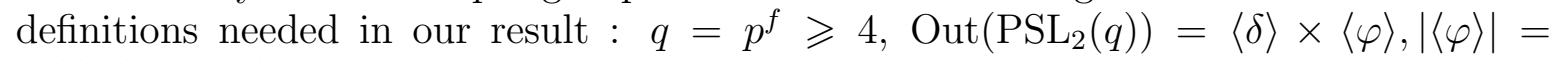
$f,|\langle\delta\rangle|=\operatorname{gcd}(2, q-1)$.

Theorem 2.4. Let $G$ be a finite almost simple group, that is $S \triangleleft G \leq A u t(S)$ for some non-abelian simple group $S$. Then $|\operatorname{cd}(G)|=5$ if and only if one of the following holds:

(a) $G \cong \operatorname{PSL}_{2}(q), q$ odd, $\operatorname{cd}(G)=\{1,(q+\epsilon) / 2, q \pm 1, q\}$,

(b) $G \cong \mathrm{PSL}_{2}\left(2^{f}\right)\langle\varphi\rangle, f>2$ prime, $\operatorname{cd}(G)=\left\{1,2^{f}-1,2^{f},\left(2^{f} \pm 1\right) f\right\}$,

(c) $G \cong \operatorname{PSL}_{2}\left(2^{f}\right)\left\langle\varphi^{f / 2}\right\rangle, f>2$ even, $\operatorname{cd}(G)=\left\{1,2^{f}, 2^{f}+1,2\left(2^{f} \pm 1\right)\right\}$, 
(d) $G \cong \mathrm{PGL}_{2}(q)\left\langle\varphi^{f / 2}\right\rangle, f>2$ even, $q$ odd, $\operatorname{cd}(G)=\{1, q, q+1,2(q \pm 1)\}$,

(e) $G \cong \mathrm{PGL}_{2}\left(3^{f}\right)\langle\varphi\rangle, f>2$ even, $\operatorname{cd}(G)=\left\{1,3^{f}, 3^{f}-1,\left(3^{f} \pm 1\right) f\right\}$,

(f) $G \cong \mathrm{PSL}_{2}(9)\langle\varphi\rangle \cong \mathrm{S}_{6}, f=2, \operatorname{cd}(G)=\{1,5,9,10,16\}$,

(g) $G \cong \operatorname{PSL}_{2}\left(5^{f}\right)\langle\varphi\rangle, f>2$ prime, $\operatorname{cd}(G)=\left\{1,5^{f},\left(5^{f}+1\right) / 2,5^{f}-1,\left(5^{f} \pm 1\right) f\right\}$,

(h) $G \cong \mathrm{PSL}_{2}(q)\left\langle\delta \varphi^{f / 2}\right\rangle, f>2$ even, $q \neq 9, p$ odd, $\operatorname{cd}(G)=\{1, q, q+1,2(q \pm 1)\}$.

Proof. First, it is well known that every simple group $S$ has a non-trivial irreducible character $\chi$ that can be extended to $\operatorname{Aut}(S)$. Suppose that $r$ is a prime that divides the character degree of $\chi$. If $r$ divides every character degree of $G$, then by Thompson's theorem [5, Corollary 12.2], $G$ has a normal $r$-complement, a contradiction.

We may thus assume that no prime divides every character degree of $G$. Since $G$ has five character degrees, there is no prime dividing four character degrees of $G$. These groups are the groups in [2, Theorem A]. It is sufficient to consider groups in case (c) since the groups in (a)-(b) and (d)-(f) can be ruled out using the character tables in the Atlas [1] and [10].

By inspecting [2, Table 1], we have our result.

\section{Main Results}

Theorem 3.1. Let $G$ be a finite almost simple group, that is $S \triangleleft G \leq$ Aut $(S)$ for some non-abelian simple group $S$. Then either $|\operatorname{cv}(G)| \geq 9$ or $|\operatorname{cv}(G)|=8$ and $G \in$ $\left\{\mathrm{PSL}_{2}(5), \mathrm{PGL}_{2}(5)\right\}$. In particular, if $|\operatorname{cd}(G)| \geq 5$, then $|\operatorname{cv}(G)| \geq 9$.

Proof. Since $G$ is non-solvable, $|\operatorname{cd}(G)| \geq 4$ by [5, Theorem 12.15]. Suppose that $|\operatorname{cd}(G)|=4$. By Theorem 2.2, it is sufficient to consider the following groups: $\mathrm{PSL}_{2}\left(2^{f}\right)$ for some $f \geq 2, \mathrm{PGL}_{2}(q)$ for some odd $q \geq 5$ and $M_{10}$. The character tables of these groups can be found in the Atlas [1], 3] and [9. In particular, $|\operatorname{cv}(G)| \geq 9$ except for $\mathrm{PSL}_{2}(5)$ and $\mathrm{PGL}_{2}(5)$. In the latter cases $|\mathrm{cv}(G)|=8$ as required.

We may assume that $|\operatorname{cd}(G)|=5$. It is sufficient to consider groups in Theorem 2.4. For cases (a) and (f), we have that $|\operatorname{cv}(G)| \geq 9$, using character tables in [3] and Atlas [1]. For cases (b), (e) and (g) we have that $C=G / \mathrm{PGL}_{2}(q)$ is cyclic of order greater than 2. Then by Lemma 2.1(b), the character table of $C$ has a column with pairwise different values. In particular, $a, b \in \operatorname{cv}(C) \backslash\{-1,0,1\}$ with $a \neq b$. Note that the Steinberg character $\psi$ of $\mathrm{PSL}_{2}(q)$ is extendible to $G$ and also $\psi(s)=-1$ for some $s \in \mathrm{PSL}_{2}(q)$ by [3, Theorems 4.7 and 4.9]. Hence $-1 \in \operatorname{cv}(G)$ and $|\operatorname{cv}(G)|=$ $|\operatorname{cd}(G) \cup\{a, b,-1,0\}| \geq 9$. Suppose that $C$ is of order 2 . Groups with this property are the ones in cases (c), (d) and (h). Consider the characters $\chi_{1}, \chi_{2} \in \operatorname{Irr}(G)$ such that $\chi_{1}(1)=2(q+1)$ and $\chi_{2}(1)=2(q-1)$. Then since $\mathrm{PGL}_{2}(q)$ has no irreducible characters of degrees $2(q \pm 1), \chi_{i}=\theta_{i 1}+\theta_{i 2}$ by [5, Theorem 6.2], where $\theta_{11}, \theta_{12}, \theta_{21}, \theta_{22} \in \operatorname{Irr}(S)$, $\theta_{11}(1)=\theta_{12}(1)=q+1$ and $\theta_{21}(1)=\theta_{22}(1)=q-1$. Using [3, Theorems 4.7 and 4.9], we have that $\theta_{11}(g)=\theta_{12}(g)=1$ and $\theta_{21}(g)=\theta_{22}(g)=-1$ for some $g \in \operatorname{PGL}_{2}(q)$. Hence $\chi_{1}(g)=2, \chi_{1}(g)=-2$ and $|\operatorname{cd}(G) \cup\{-2,-1,0,2\}| \geq 9$.

Going forward we may assume that $|\operatorname{cd}(G)| \geq 6$. Suppose that $|\operatorname{cd}(G)| \geq 6$ and $S \cong \mathrm{PSL}_{2}(q)$. We may assume that $G$ is not isomorphic to $\operatorname{PSL}_{2}\left(2^{f}\right)$ with $f \geq 2$, $\mathrm{PSL}_{2}\left(p^{f}\right)$ with $p$ odd or $\mathrm{PGL}_{2}\left(p^{f}\right)$ with $p$ odd. Let $H \in\left\{\mathrm{PSL}_{2}\left(2^{f}\right), f \geq 2\right\} \cup\left\{\mathrm{PSL}_{2}(q)\right.$, $q$ odd $\} \cup\left\{\mathrm{PGL}_{2}(q), q\right.$ odd $\}$. If $G \cong H \mathrm{C}_{r}$, where $r \mid f, r>2$. Then arguing as above, we have that $a, b \in \operatorname{cv}\left(\mathrm{C}_{r}\right) \backslash\{-1,1\}$ and $|\operatorname{cv}(G)|=|\operatorname{cd}(G) \cup\{-1,0, a, b\}| \geq 10$. If $G \cong H \mathrm{C}_{2}, 2 \mid f$. Then either $2(q-1)$ or $2(q+1)$ is a character degree of $G$. Arguing as above, we have that either $-2 \in \mathrm{cv}(\mathrm{C})$ or $2 \in \mathrm{cv}(\mathrm{C})$. Since $-1,0 \in \mathrm{cv}(G)$ and $|\operatorname{cd}(G)| \geq 6$ we have that $|\operatorname{cv}(G)| \geq 9$. 
If $S \cong \mathrm{PSL}_{3}(4)$, then the result follows using the character table in the Atlas [1]. Suppose that $S \cong 2 \mathrm{~B}_{2}\left(2^{f}\right)$, where $2^{f}=2^{2 m+1}$ for some integer $m \geq 1$. If $G \cong{ }^{2} \mathrm{~B}_{2}\left(2^{f}\right)$, it follows that $|\operatorname{cv}(G)| \geq 9$ by the character table in [10, Theorem 13]. We may assume that $S<G<\operatorname{Aut}(S)$. Then $C=G / S$ is cyclic of odd order. Using the argument as in the case of $\operatorname{PSL}_{2}(q)$ above, we have that $a, b \in \operatorname{cv}(C) \backslash\{-1,1\}$. Hence $|\operatorname{cv}(G)| \geq$ $|\operatorname{cd}(G) \cup\{a, b, 0\}|=9$.

If $|\operatorname{cd}(G)| \geq 7$, then by column orthogonality, there is a character value which is a negative number and so $|\operatorname{cv}(G)| \geq 9$. This concludes our proof.

Proof of Theorem 1.1. We prove our result using induction on $|G|$. Since $|\operatorname{cv}(G)|<$ 8, every homomorphic image of $G$ has the same property. Let $N$ be a minimal normal subgroup of $G$. If another such minimal normal subgroup, say $M$, exists then $G$ is the subdirect product of solvable groups $G / N$ and $G / M$, and so is solvable. This implies that $N$ is unique and non-abelian. Hence $G$ is almost simple group. The result follows by Theorem 3.1 .

Before we prove Theorem [1.2, we recall a definition. If $N \triangleleft G$, then $\operatorname{Irr}(G \mid N)=\{\chi \in$ $\operatorname{Irr}(G) \mid N \nsubseteq \operatorname{ker} \chi\}$ and $\operatorname{cd}(G \mid N)=\{\chi(1) \mid \chi \in \operatorname{Irr}(G \mid N)\}$.

Proof of Theorem 1.2. Since $G$ is non-solvable, $|\operatorname{cd}(G)| \geq 4$. If $5 \leq|\operatorname{cd}(G)| \leq 6$, then by Theorem [2.3, $G^{\prime} / L$ is isomorphic to $\mathrm{PSL}_{2}\left(p^{f}\right), p^{f} \geq 4, \mathrm{PSL}_{3}(4)$ or ${ }^{2} \mathrm{~B}_{2}\left(2^{f}\right)$, where $2^{f}=2^{2 m+1}$ for some integer $m \geq 1$, where $L$ is the solvable radical of $G$. Specifically, $G / L$ is an almost simple group. Using Theorem 3.1 , $|\operatorname{cv}(G)| \geq|\operatorname{cv}(G / L)| \geq$ 9.

If $|\operatorname{cd}(G)|=4$, then using Theorem 2.2 and Theorem 3.1, it is sufficient to consider $G \cong \mathrm{PSL}_{2}(5) \times A$ for some abelian subgroup $A$ or $G / C \cong \mathrm{PGL}_{2}(5)$, where $C=\mathrm{C}_{G}(U)$, $U=\mathrm{PSL}_{2}(5)$ or $\mathrm{SL}_{2}(5)$ with $C \leq \mathbf{Z}(G)$ and $U$ is a normal subgroup of $G$. Suppose that $G \cong \mathrm{PSL}_{2}(5) \times A$ with non-trivial $A$. Consider $\theta \in \operatorname{Irr}\left(\mathrm{PSL}_{2}(5)\right)$ such that $\theta(1)=5$ and $\phi \in \operatorname{Irr}(A)$ such that $\phi(g)=k<0$. Then $\chi(1 \times g)=\theta(1) \times \phi(g)=5 k<0$ and $|\operatorname{cv}(G)| \geq 9$. Suppose that $G$ is a group in the latter case. We want to show that $C=1$. Suppose that $C \neq 1$. Let $\mathbf{Z}(G) U=N$ and note that $\operatorname{cd}(G \mid N) \geq 3$ by [6, Theorem B]. Then there is a $\chi \in \operatorname{Irr}(G)$ such that $\chi(1)=m \in \operatorname{cd}(G \mid N) \backslash\{1,2\}$. For $1 \neq z \in \mathbf{Z}(G)$, we have that $\chi(z)=-m$. This means that $|\operatorname{cv}(G)| \geq\left|\mathrm{PGL}_{2}(5) \cup\{-m\}\right|=9$. Therefore $G=\mathrm{PGL}_{2}(5)$ and that concludes our argument.

\section{ACKNOWLEDGEMENTS}

The author would like to thank the reviewer for their comments which improved the presentation of the article.

\section{REFERENCES}

[1] J. H. Conway, R. T. Curtis, S. P. Norton, R. A. Parker and R. A. Wilson, Atlas of Finite Groups, Clarendon Press, 1985.

[2] M. Ghaffarzadeh, M. Ghasemi, M. L. Lewis, H. P. Tong-Viet, Nonsolvable groups with no prime dividing four character degrees, Algebr. Represent. Theory 20 (2017) 547-567.

[3] K. E. Gehles, Ordinary characters of finite special linear groups, MSc thesis, University of St Andrews, 2002.

[4] L. He and G. Zhu, Nonsolvable normal subgroups and irreducible character degrees, J. Algebra 372 (2012) 68-84.

[5] I. M. Isaacs, Character Theory of Finite Groups, Amer. Math. Soc., Providence, Rhode Island, 2006. 
[6] I. M. Isaacs, G. Knutson, Irreducible character degrees and normal subgroups, J. Algebra 199 (1998) 302-326.

[7] G. Malle and A. Moretó, Nonsolvable groups with few character degrees, J. Algebra 294 (2005) $117-126$.

[8] T. Sakurai, Finite groups with very few character values, Comm. Algebra 49 (2021) 658-661.

[9] R. Steinberg, The representations of $\mathrm{GL}(3, q), \mathrm{GL}(4, q), \operatorname{PGL}(3, q)$ and $\mathrm{PGL}(4, q)$, Canad. J. Math. 3 (1951) 225-235.

[10] M. Suzuki, On a class of doubly transitive groups, Ann. of Math. 75 (1962) 105-145.

Department of Mathematics and Applied Mathematics, University of Pretoria, Private Bag X20, Hatfield, Pretoria 0028, South Africa

Email address: sesuai.madanha@up.ac.za 